ARTICLE

\title{
Scalable fabrication of a hybrid field-effect and acousto-electric device by direct growth of monolayer $\mathrm{MoS}_{2} / \mathrm{LiNbO}_{3}$
}

Edwin Preciado ${ }^{1, \star}$, Florian J.R. Schülein ${ }^{2,3, \star}$, Ariana E. Nguyen ${ }^{1}$, David Barroso ${ }^{1}$, Miguel Isarraraz ${ }^{1}$, Gretel von Son $^{1}$, I-Hsi Lu', Wladislaw Michailow², Benjamin Möller², Velveth Klee1, John Mann ${ }^{4}$, Achim Wixforth ${ }^{2,3,5}$, Ludwig Bartels ${ }^{1} \&$ Hubert J. Krenner ${ }^{2,3,5}$

Lithium niobate is the archetypical ferroelectric material and the substrate of choice for numerous applications including surface acoustic wave radio frequencies devices and integrated optics. It offers a unique combination of substantial piezoelectric and birefringent properties, yet its lack of optical activity and semiconducting transport hamper application in optoelectronics. Here we fabricate and characterize a hybrid $\mathrm{MoS}_{2} / \mathrm{LiNbO}_{3}$ acousto-electric device via a scalable route that uses millimetre-scale direct chemical vapour deposition of $\mathrm{MoS}_{2}$ followed by lithographic definition of a field-effect transistor structure on top. The prototypical device exhibits electrical characteristics competitive with $\mathrm{MoS}_{2}$ devices on silicon. Surface acoustic waves excited on the substrate can manipulate and probe the electrical transport in the monolayer device in a contact-free manner. We realize both a sound-driven battery and an acoustic photodetector. Our findings open directions to non-invasive investigation of electrical properties of monolayer films.

\footnotetext{
${ }^{1}$ Chemistry, Materials Science \& Engineering and Electrical Engineering, University of California, Riverside, California 92521, USA. ${ }^{2}$ Lehrstuhl für Experimentalphysik 1 and Augsburg Centre for Innovative Technologies (ACIT), Universität Augsburg, Universitätsstrasse 1, Augsburg 86159, Germany. ${ }^{3}$ Nanosystems Initiative Munich (NIM), Schellingstrasse 4, München 80799, Germany. ${ }^{4}$ Department of Physics, Pepperdine University, 24255 Pacific Coast Highway, Malibu, California 90263, USA. ${ }^{5}$ Center for NanoScience (CeNS), Ludwig-Maximilians-Universität München, Geschwister-Scholl-Platz 1, München 80539, Germany. ${ }^{*}$ These authors contributed equally to this work. Correspondence and requests for materials should be addressed to L.B. (email: ludwig.bartels@ucr.edu) or to H.J.K. (email: hubert.krenner@physik.uni-augsburg.de).
} 
$\mathrm{T}$ hrough a combination of substantial piezoelectric and birefringent properties, $\mathrm{LiNbO}_{3}$ offers pronounced electrooptic, acousto-optic and acousto-electric activities ${ }^{1}$ that render it the material of choice for numerous applications in radio frequency (RF) signal processing ${ }^{2,3}$ and passive integrated optics' $^{4} \mathrm{LiNbO}_{3}$, the 'silicon of photonics ${ }^{5,6}$, exhibits a large, $\sim 3.95 \mathrm{eV}$ indirect bandgap ${ }^{7}$. For application as an optical sensor, hybridization with a lower bandgap material is of key relevance. Here we report millimetre-scale direct chemical vapour deposition of monolayer $\mathrm{MoS}_{2}$ onto $128^{\circ} \mathrm{YX}$-cut $\mathrm{LiNbO}_{3}$. Fieldeffect transistors (FETs) fabricated on these films exhibit characteristics competitive with established transition metal dichalcogenide (TMD) devices on silicon ${ }^{8-11}$. The acoustoelectric activity of the $\mathrm{LiNbO}_{3}$ substrate permits concomitant control and measurement of the systems electronic and optical properties in a contact-free manner. In our hybrid device, surface acoustic waves (SAWs) excited directly on the $\mathrm{LiNbO}_{3}$ substrate induce a strong acousto-electric effect and sense remotely the photoconductance of an TMD monolayer. SAW photoconductance spectroscopy can be performed at any point along the propagation path of the wave that extends on the millimetre length scale of a chip. This is in strong contrast to contact-based transport measurements, for which only the sample area between the contacts can be probed.

TMD films have attracted considerable attention for optoelectronic applications because of their direct-bandgap-semiconducting property at the single-layer limit ${ }^{12,13}$. Offering a tunable bandgap of 1.1-1.9 eV $\left(\mathrm{MoTe}_{2}\right.$ to $\left.\mathrm{WS}_{2}\right)$, these two-dimensional (2D) semiconductors can complement the properties of graphene through their strong photoluminescence (PL), significant spinorbit coupling ${ }^{14,15}$, and ensuing valleytronics physics ${ }^{16,17}$. Numerous reports have shown functional FET devices of $\mathrm{MoS}_{2}$ and other TMDs ${ }^{8-11}$; prototypical TMD FETs have been applied for gas sensing ${ }^{18}$, extended to ferroelectric gating ${ }^{19,20}$ and were used to construct a memory element ${ }^{21,22}$. So far, most investigations have focused on conventional semiconductor platforms, typically utilizing TMD material exfoliated onto $\mathrm{SiO}_{2} / \mathrm{Si}$ substrates. A range of chemical vapour deposition-based growth methods has been developed for TMD films on different substrates, including $\mathrm{SiO}_{2}$, sapphire, and graphene ${ }^{23-30}$. $\mathrm{LiNbO}_{3}$ is routinely used in numerous commercial SAW devices, ranging from RF filters to wirelessly interrogated and identity-tagged devices, as well as in microfluidics. In addition, $\mathrm{LiNbO}_{3}$ is the material of choice for integrated photonic devices that harness its native nonlinear optical properties for classical and quantum communication. Hence, the hybridization of TMDs with $128^{\circ} \mathrm{YX}-$ cut $\mathrm{LiNbO}_{3}$ is a key technological advancement.

Acousto-electric spectroscopy has a long-standing tradition in probing and controlling solid-state materials ${ }^{31,32}$. SAWs in particular offer a versatile approach since these nanoscale sound waves can be excited and detected all-electrically on a chip. SAW spectroscopy has been performed on bulk semiconductors and their heterostructures even in the quantum regime ${ }^{33-35}$. For more than 15 years, semiconductor- $\mathrm{LiNbO}_{3}$ hybrids have been realized by epitaxial lift-off and transfer onto the $\mathrm{LiNbO}_{3}$ substrate ${ }^{36}$. Recently, similar hybrids have been reported for graphene ${ }^{37-40}$. These applications have in common that the semiconducting layer is loosely attached to the substrate via weak van der Waals' interactions. This interaction preserves contact-free acousto-electric access but its ability to offer acoustomechanical couplings has not been determined.

A particularly exciting aspect of transport measurements using SAWs is their inherently contact-free nature combined with their sensitivity in the limit of low conductivity. The transport properties reported for TMD materials, such as transconductance, carrier mobility and susceptibility to gating, vary widely even for measurements on the same TMD material. From the perspective of technological application, this is very undesirable. In particular, charge transfer at the interface between metal contacts and the 2D TMD films, resulting in band shifting/bending analogue to the formation of a Schottky barrier, has been reported to affect transport measurements ${ }^{41-43}$. SAW-based devices will ultimately have the power to provide entirely contact-free measurements, thus opening a new avenue to shed light on this current issue.

Here we report on two technological advancements: the scalable fabrication of a hybrid $\mathrm{MoS}_{2} / \mathrm{LiNbO}_{3}$ FET-electroacoustic device that combines FET functionality with response to SAWs and the cross-validation of the respective signals; we demonstrate the versatility and power of this approach by measurement of the photoconductivity of a single-layer $\mathrm{MoS}_{2}$ film.

\section{Results}

Device layout and characteristics. A schematic of our hybrid device is shown in Fig. 1a and the fabrication procedure is summarized in the Methods section. The device consists of two components: (i) a SAW delay line formed by a pair of interdigital transducers (IDTs) and (ii) a monolayer $\mathrm{MoS}_{2}$-FET centred in between the two IDTs. This configuration enables us to probe and manipulate the electrical characteristics of the FET by exciting and detecting SAWs interacting with carriers in the $\mathrm{MoS}_{2}$. IDTs are used for all-electrical excitation and detection of SAWs. On $\mathrm{LiNbO}_{3}$, SAWs propagate at a velocity $v_{\mathrm{SAW}}=3,980 \mathrm{~m} \mathrm{~s}^{-1}$; our IDTs are designed for a frequency of $f_{\mathrm{SAW}}=160 \mathrm{MHz}$ corresponding to a design wavelength $\lambda_{\mathrm{SAW}}=25 \mu \mathrm{m}$. Their arrangement allows measurement of the scattering parameter, $S_{21}$, that is, the SAW transmission from one IDT to the other. In Fig. $1 \mathrm{~b}$ we plot $S_{21}$ as a function of the RF signal applied to the sending IDT. In this trace, the delay line resonance frequency is resolved as a $40-\mathrm{dB}$ high transmission maximum very close to the nominal design frequency of $f_{\mathrm{SAW}}=160 \mathrm{MHz}$. This $\mathrm{RF}$ characterization demonstrates high efficiency generation, transmission and detection of SAWs on the $\mathrm{LiNbO}_{3}$ host substrate even after its exposure to the $\mathrm{MoS}_{2}$ growth conditions. FET fabrication was performed on a monolayer region of the as-grown $\mathrm{MoS}_{2}$ film; its single-layer thickness was validated by scanning PL spectroscopy. An overview map demonstrating millimetre-scale growth of monolayer $\mathrm{MoS}_{2}$ onto $128^{\circ} \mathrm{YX}$-cut $\mathrm{LiNbO}_{3}$ is presented in Supplementary Fig. 1. After PL characterization, a 4-terminal $\mathrm{MoS}_{2}$ FET is monolithically defined on the $\mathrm{LiNbO}_{3}$ substrate in the region of maximum emission of monolayer $\mathrm{MoS}_{2}$. The FET is fabricated to be located in between a pair of IDTs. Figure 1c shows a $400 \mu \mathrm{m} \times 350 \mu \mathrm{m}$ spatial map of the characteristic monolayer $\mathrm{MoS}_{2} \mathrm{PL}$ emission in the channel region ${ }^{12,13}$ of the FET. The PL intensity is encoded in colour scale with red/dark regions corresponding to high/low count rates, respectively. PL mapping is used to determine the extent of the monolayer film before device fabrication and it confirms the presence of a monolayer $\mathrm{MoS}_{2}$ channel in the completed FET. Intensity variations are likely due to variations in the film domain size as shown in ref. 25. In all other areas, the $\mathrm{MoS}_{2}$ film was selectively removed to avoid any signal contributions from these regions. The four vertical lines indicate reflection from the $\mathrm{Au}$ contact lines; the four diffuse spots close to the corners of the panel represent gold alignment marks used during fabrication of the FET channel. The upper and lower boundaries of the FET channel in Fig. 1c are aligned with the SAW propagation path. Thus, we ensure tight correspondence of the electrically and acoustically addressed film area. The $\mathrm{LiNbO}_{3}$ substrates serves as the $\left(\epsilon_{\mathrm{r}} \sim 50\right)$ dielectric for back gating to ensure full FET 
a

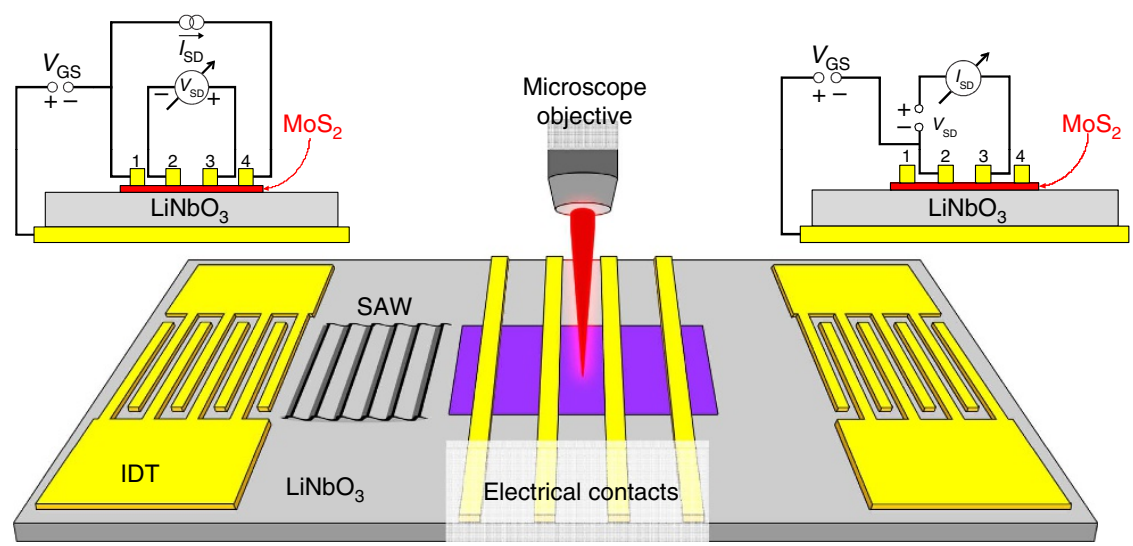

b

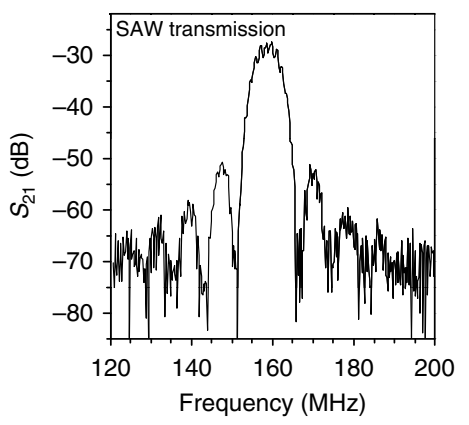

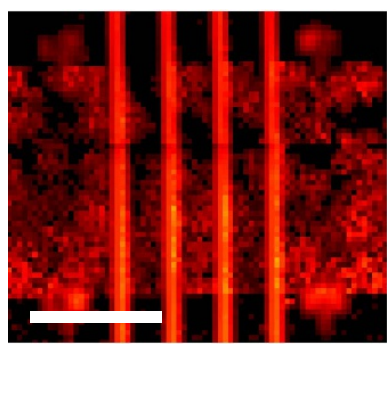

d

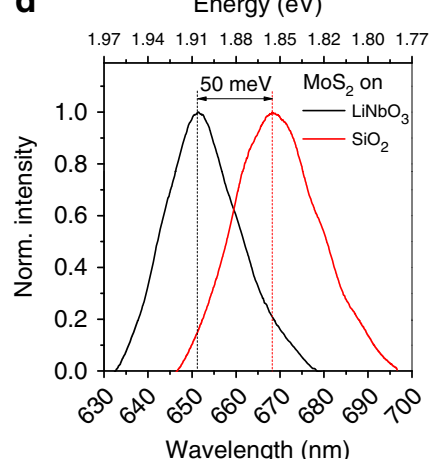

Figure 1 | Sample. (a) Schematic representation of our hybrid $\mathrm{MoS}_{2} / \mathrm{LiNbO}_{3}$ device. Four Ti/Au electrodes form the contacts of a FET fabricated on chemical vapour deposition-grown $\mathrm{MoS}_{2}$. Two opposing, non-impedance-matched IDTs are used to excite SAWs propagating across the MoS 2 FET. The insets show the electrical wiring configurations for 4-point (left) and 2-point (right) measurements. The sample was excited optically using a $\times 50$ microscope objective with a numerical aperture (NA) of 0.55. (b) SAW transmission between the IDTs across the FET device shows a pronounced $40 \mathrm{~dB}$ maximum at the design frequency $f_{\mathrm{SAW}}=160 \mathrm{MHz}$ of the 5.4-mm long delay line. (c) PL map of the active FET region (scale bar, $100 \mu \mathrm{m}$ ). Monolayer MoS 2 PL intensity (colour coded: red high intensity, black low intensity) is detected only in the channel region. Reflection from the FET contacts and alignment marks is clearly visible. (d) Comparison of single-point $\mathrm{PL}$ spectra obtained on $\mathrm{SiO}_{2}$ (red) and our $128^{\circ} \mathrm{YX}$-cut $\mathrm{LiNbO}_{3}$ substrate (black) reveals a blueshift attributed to compression of the $\mathrm{MoS}_{2}$ film. Norm., normalized.

operation and we provide full electrical characterization of this device. We show the respective wiring diagrams for 4- and 2point configuration as insets in Fig. 1a.

In Fig. 1d, we compare normalized PL spectra from $\mathrm{MoS}_{2}$ films grown under nominally identical conditions on $\mathrm{LiNbO}_{3}$ (black) and on a $\mathrm{SiO}_{2} / \mathrm{Si}$ reference substrate (red). For both samples, a PL peak is clearly resolved, corroborating the monolayer nature of the $\mathrm{MoS}_{2}$ (Supplementary Fig. 2 also provides Raman spectroscopy). However, $\mathrm{MoS}_{2}$ grown on $128^{\circ} \mathrm{YX}$-cut $\mathrm{LiNbO}_{3}$ yields $\mathrm{PL}$ signal at photon energies larger by $40-50 \mathrm{meV}$; we attribute this shift to the fivefold larger thermal expansion coefficient of $\mathrm{LiNbO}_{3}$ (ref. 44) of $\alpha_{\mathrm{LiNbO}_{3}}=12.6 \times 10^{-6} \mathrm{~K}^{-1}$ compared with that of $\mathrm{Si}$ (ref. 45) of $\alpha_{\mathrm{Si}}=2.6 \times 10^{-6} \mathrm{~K}^{-1}$ near room temperature. These dissimilar values cause a net relative compressive strain of $0.35 \%$ for the $\mathrm{MoS}_{2}$ film on $\mathrm{LiNbO}_{3}$ during cool-down from growth temperatures. Compressive strain is expected to give rise to a blueshift of the PL emission. Extrapolating the data on uniaxial tensile stress reported in ref. 46 of $\Delta E=60-70 \mathrm{meV}$ per $1 \%$ strain, we expect a blueshift of $\sim 23 \mathrm{meV}$, about $1 / 2$ of the observed blueshift. Using the value of $\Delta E=300 \mathrm{meV}$ per $1 \%$ strain reported by Hui et al. ${ }^{47}$ for compressive strain in trilayer $\mathrm{MoS}_{2}$, the expected blueshift amounts to $105 \mathrm{meV}$, which is larger than the value observed here. Thus, our observation of a blueshift is compatible in magnitude with recent work and corroborates a rigid connection of the $\mathrm{MoS}_{2}$ film to the $\mathrm{LiNbO}_{3}$ substrate, a precondition for maximum interaction with the SAW.
FET characterization. We expect our hybrid device to exhibit FET properties. Owing to the very large dielectric constant $\varepsilon_{\mathrm{r}} \sim 50$ for this cut of $\mathrm{LiNbO}_{3}$, moderate electric fields $D=\varepsilon_{\mathrm{r}} V_{\mathrm{GS}} /$ $d_{\text {sub }}= \pm 40 \mathrm{kV} \mathrm{cm}^{-1}$ can be achieved by applying $V_{\mathrm{GS}}= \pm 40 \mathrm{~V}$ between the $\mathrm{LiNbO}_{3}$ backside (thickness $d_{\text {sub }}=500 \mu \mathrm{m}$ ) and the $\mathrm{MoS}_{2}$ layer. We test FET operation of our hybrid device by measurement of its transport characteristics as a function of a back gate voltage $\left(V_{\mathrm{GS}}\right)$ across the $\mathrm{LiNbO}_{3}$ substrate. In Fig. 2a, we plot a set of output characteristics $\left(I_{\mathrm{SD}}\right.$ versus $\left.V_{\mathrm{SD}}\right)$, recorded in 4-point configuration, for different back gate voltages ranging between $V_{\mathrm{GS}}= \pm 40 \mathrm{~V}$. As $V_{\mathrm{GS}}$ is tuned from negative to positive polarity, we observe the expected reduction of the sheet resistance due to accumulation of electrons in the $\mathrm{MoS}_{2}$ monolayer. This observation clearly demonstrates FET operation in our hybrid device in the linear regime and the formation of an $n$-type transport channel, which is in agreement with prior work ${ }^{8-11}$. Transfer characteristics $\left(I_{\mathrm{SD}}\right.$ versus $V_{\mathrm{GS}}$ ) recorded in 2-point configuration are plotted in Fig. $2 \mathrm{~b}$ for different $V_{\mathrm{SD}}$. Again, the increase of $I_{\mathrm{SD}}$ as $V_{\mathrm{GS}}$ is tuned to positive bias at constant $V_{\mathrm{SD}}$ is consistent with $n$-type character. We note that in the data, a small leakage current through the substrate was subtracted for clarity; Supplementary Fig. 3a contains the graphs before subtraction of gate leakage. The transfer characteristics exhibit a small hysteresis, which may arise from poling effects of $\mathrm{LiNbO}_{3}$ at the interface to the $\mathrm{MoS}_{2}$ layer ${ }^{19}$. We note that $\mathrm{MoS}_{2}$-based FETs on dielectric $\mathrm{Si} / \mathrm{SiO}_{2}$ substrates have been found to be sensitive to 

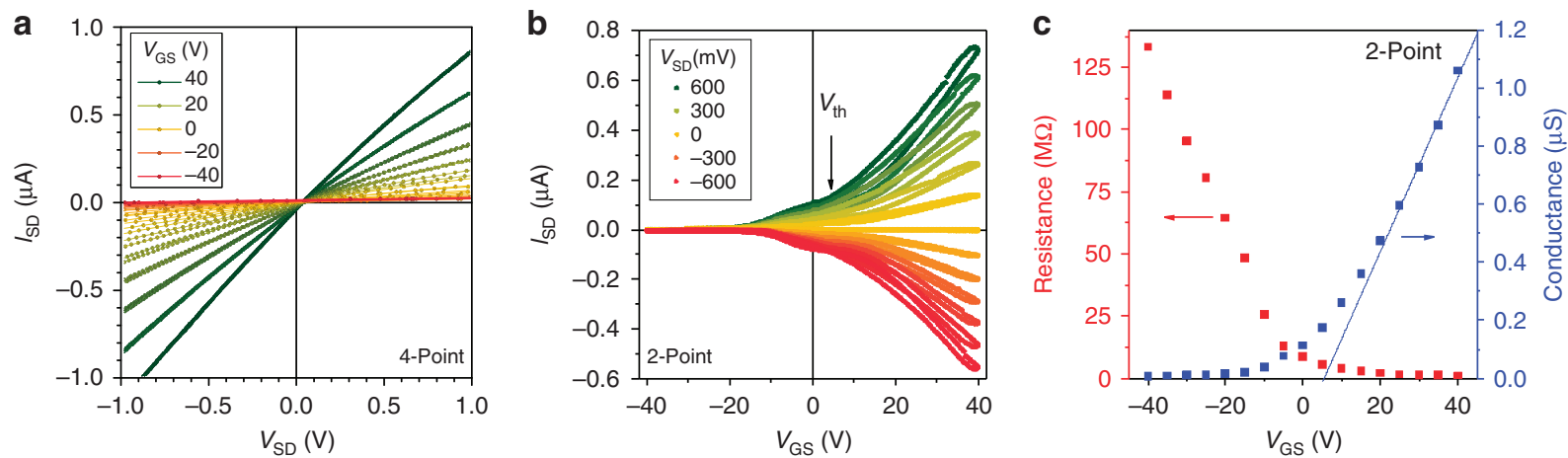

Figure 2 | FET operation of hybrid $\mathbf{M o S}_{2} / \mathbf{L i N b O}_{3}$ device. (a) Output characteristics ( $I_{S D}$ versus $\left.V_{S D}\right)$ for different gate voltages $V_{G S}$ recorded in 4-point configuration. For large negative $V_{G S}$, the device is weakly conducting; an $n$-type channel is formed for positive $V_{G S}$. (b) Transfer characteristics ( $I_{\mathrm{SD}}$ versus $\left.V_{\mathrm{GS}}\right)$ for different source-drain voltages $V_{\mathrm{SD}}$ recorded in 2-point configuration shows pronounced increase of $\left|I_{\mathrm{SD}}\right|$ at positive $V_{\mathrm{GS}}$ due to formation of an $n$-type channel. (c) Channel resistance (red) and conductance (blue) as a function of $V_{G S}$ extracted from 2-point output characteristics at $V_{\mathrm{SD}}=0$. For positive $V_{\mathrm{GS}}$ a linear fit indicates a mobility $\mu_{\mathrm{FE}}=33 \pm 5 \mathrm{~cm}^{2} \mathrm{Vs}^{-1}$ and a threshold voltage $V_{\text {th }}=5.5 \pm 1.5 \mathrm{~V}$. The latter agrees well with that derived from the data in $\mathbf{b}$, as summarized in Table 1.

the local environment and, in particular, to the surrounding gas atmosphere $^{18}$. Adsorption and desorption of impinging gas atoms and molecules have been suggested as an origin of an hysteretic $I-V$ characteristics ${ }^{10}$. From the turn-on behaviour, we are able to derive the threshold voltage $V_{\text {th }}$, marked in Fig. 2 b. From this analysis, we can determine the field-effect mobility in our device given by $\mu_{\mathrm{FE}}=\frac{L \times d_{\mathrm{sub}}}{W \epsilon_{\mathrm{r}} \epsilon_{0}} \times \frac{1}{V_{\mathrm{SD}}} \times \frac{\mathrm{d} I_{\mathrm{SD}}}{\mathrm{d} V_{\mathrm{GS}}}$, with $L$ and $W$ being the length and width of the channel, respectively and $d_{\text {sub }}$ denotes the thickness of the $\mathrm{LiNbO}_{3}$ substrate. An example analysis is presented in Supplementary Fig. $3 \mathrm{~b}$, and the $V_{\mathrm{SD}}$ dependence of the values $V_{\text {th }}$ and $\mu_{\mathrm{FE}}$ are presented in Supplementary Fig. $3 c$ and $d$, respectively. From the output characteristics set, we are able to extract the channel's electrical properties as a function of $V_{\mathrm{Gs}}$. Figure $2 \mathrm{c}$ depicts the channel resistance and conductance extracted from 2-point output characteristics evaluated at $V_{\mathrm{DS}}=0$ and plotted versus $V_{\mathrm{GS}}$. Moreover, from a linear fit of the conductance for $V_{\mathrm{GS}}>20 \mathrm{~V}$, we can determine $\mu_{\mathrm{FE}}$ and $V_{\mathrm{th}}$ from the slope and from the intersection at $I_{\mathrm{SD}}=0$, respectively. We performed an analogous analysis on the 4-point output characteristics that is presented in Supplementary Fig. 4. Table 1 summarizes the results for $\mu_{\mathrm{FE}}$ and $V_{\mathrm{th}}$; the error estimates originate from the accuracy of the linear fit and from the s.d. of $12 V_{\mathrm{GS}}$ up- and down-sweeps for output and transfer characteristics, respectively. The values for $\mu_{\mathrm{FE}}$ and $V_{\text {th }}$ derived from these independent sets of data are in good agreement. We note that the values obtained on our highly piezoelectric architecture are competitive with back-gated devices fabricated by exfoliation on the $\mathrm{SiO}_{2} / \mathrm{Si}$ platform ${ }^{48}$.

Acousto-electric effect. Having established the electrical functionality of our hybrid device, we validate its acousto-electric transport properties. Acousto-electric effects are expected within the transmission band of the SAW delay line. The frequency dependence of the corresponding scattering parameter $S_{21}$ is plotted in Fig. 3a. First, we measure the short-circuit $\left(V_{\mathrm{SD}}=0\right)$ acousto-electric current (AEC; Fig. 3b) in a two-probe configuration as a function of an RF signal of varying frequency and power $P_{\mathrm{RF}}$ applied to each of the two IDTs. For a forward propagating SAW excited by constant $P_{\mathrm{RF}}$, we observe that the AEC exhibits a characteristic frequency dependence. This dependence faithfully reproduces the $S_{21}$ data. As the propagation direction of the SAW is reversed, the polarity of the AEC reverses: this finding indicates that the propagation direction of the SAW determines
Table 1 | Field-effect mobility and threshold voltage of our FET device determined by different techniques.

\begin{tabular}{lccc} 
& $\begin{array}{c}\text { 2-Point output } \\
\text { characteristics } \\
\left(\boldsymbol{V}_{\mathbf{S D}}=\mathbf{0}\right)\end{array}$ & $\begin{array}{c}\text { 4-Point output } \\
\text { characteristics } \\
\left(\boldsymbol{V}_{\mathbf{S D}}=\mathbf{0}\right)\end{array}$ & $\begin{array}{c}\text { 2-Point transfer } \\
\text { characteristics }\end{array}$ \\
\hline$\mu_{\mathrm{FE}}\left(\mathrm{cm}^{2} \mathrm{~V}^{-1} \mathrm{~s}^{-1}\right)$ & $33 \pm 5$ & $43 \pm 5$ & $37 \pm 5$ \\
$V_{\mathrm{th}}(\mathrm{V})$ & $5.5 \pm 1.5$ & $5.2 \pm 1.5$ & $5.8 \pm 2.5$ \\
\hline $\mathrm{FET}$, Field-effect transistor. & & & \\
\hline
\end{tabular}

the direction of the carrier flow between the two contacts (that is, momentum transfer between the SAW and the mobile carriers in the $\mathrm{MoS}_{2}$ film). The observed polarities provide an independent verification of $n$-type majority charge carriers in the $\mathrm{MoS}_{2}$ film. We note that the different AEC levels measured for the two IDTs at constant $P_{\mathrm{RF}}$ arise from a combination of variations in their absolute conversion efficiencies, different distances from the location of our measurements and different SAW attenuation along the propagation path. The lower amplitudes of the AEC compared with reports on graphene on $\mathrm{LiNbO}_{3}$ (refs 37,38) are expected due to the lower carrier concentrations in our $\mathrm{MoS}_{2}$ films compared with zero-bandgap graphene.

Second, we explore the $P_{\mathrm{RF}}$ power dependence of the acoustoelectric effect for both SAW directions in a complementary experiment by measuring the open-circuit voltage in a 4-point configuration (Fig. 1b) with open connection to the back contact. Here the total current between the two outer contacts is set to $I_{\mathrm{SD}}=0$ and the acousto-electric voltage $(\mathrm{AEV})$ is picked up between the two inner contacts. In Fig. $3 c$, we plot the measured $\mathrm{AEV}$ as a function of $P_{\mathrm{RF}}$ (in $\mathrm{mW}$ ) applied for a forward (black) and reverse (red) propagating SAW. We observe the expected linear power dependence of the acousto-electric effect. We note that the AEC and voltage represent sound-driven constant current and voltage sources, respectively. Such an 'acoustic battery' is remotely driven by the SAW.

Contact-free photoconductivity probe. Having established the functionality of our hybrid device, we proceed by applying it to the measurement of the photoconductivity of the $\mathrm{MoS}_{2}$ layer. $\mathrm{MoS}_{2}$ single-layer material exhibits a pronounced photoconductive response to optical (above bandgap) irradiation ${ }^{49-51}$. 

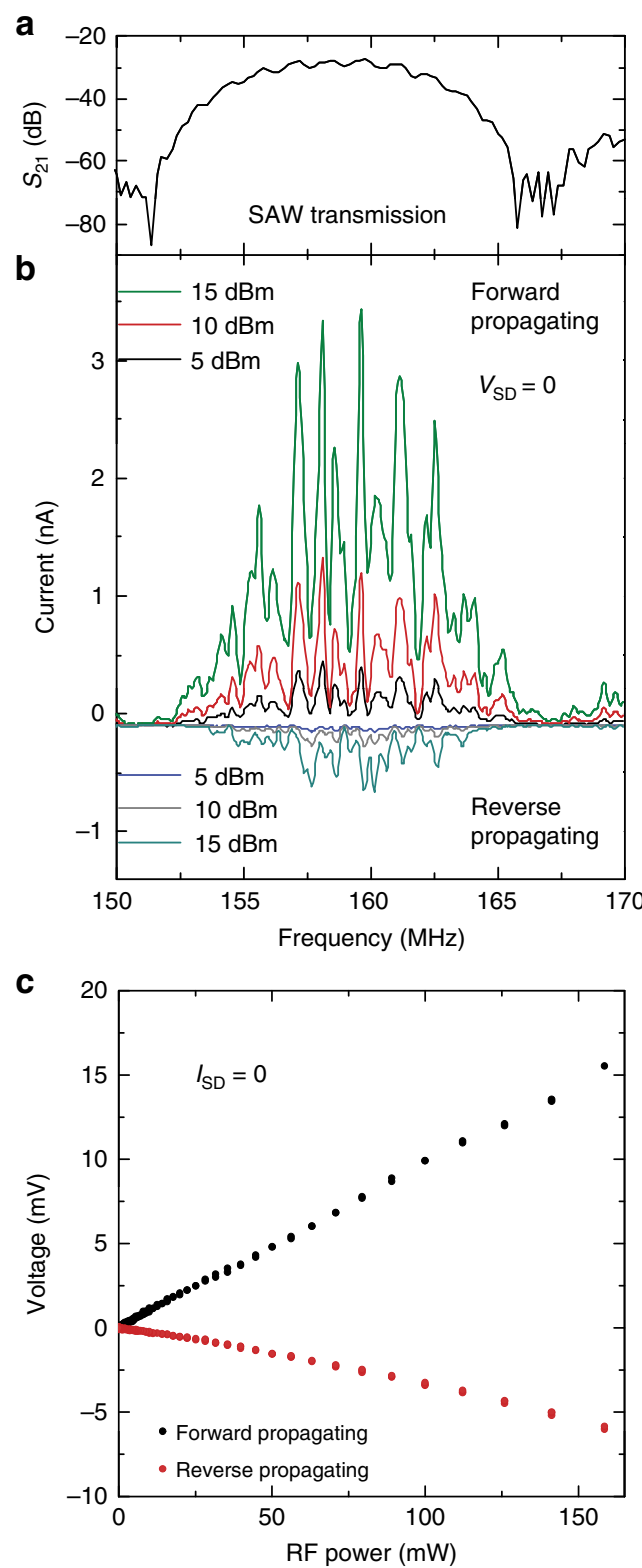

Figure 3 | Acousto-electric spectroscopy. (a) Frequency band of the SAW transmission between IDTs plotted as the scattering parameter $S_{21}$. (b) AEC as a function of RF applied to the IDTs for different RF power levels $P_{\mathrm{RF}}$ Current measurements were performed in a 2-point short-circuit $\left(V_{S D}=0\right)$ configuration. The forward and reverse propagating SAWs were excited by either of the two opposing IDTs. They yield AECs of opposite sign. (c) AEV as a function of $P_{\mathrm{RF}}$ measured in 4-point, open-circuit configuration $\left(I_{\mathrm{SD}}=0\right)$. For both SAW propagation directions, the expected linear dependence is well reproduced. The signs of the AECs and voltages correspond to $n$-type conductivity of the film.

SAWs provide an extremely sensitive and fast conductivity $(\sigma)$ probe and are particular suitable to the characterization of poorly conductive films ${ }^{52}$. The SAW attenuation coefficient is given by:

$$
\Gamma=\frac{K_{\mathrm{eff}}^{2} \times k_{\mathrm{SAW}}}{2} \times \frac{\sigma / \sigma_{\mathrm{m}}}{1+\left(\sigma / \sigma_{\mathrm{m}}\right)^{2}}
$$

In this expression, $K_{\text {eff }}^{2}=0.056$ is the electromechanical coupling efficiency, $k_{\mathrm{SAW}}$ is the SAW wave vector and $\sigma_{\mathrm{m}}=v_{\mathrm{SAW}} \times \epsilon_{0}\left(1+\epsilon_{\mathrm{r}}\right)=1.8 \times 10^{-6} \frac{1}{\Omega_{\square}} \quad$ is the characteristic sheet conductivity. This value corresponds to a characteristic channel conductance of $G_{\mathrm{m}}=\sigma_{\mathrm{m}} \times \frac{W}{L} \sim 18.5 \mu \mathrm{S}$ in our device. This value is larger than the measured channel conductance $G<1.2 \mu \mathrm{S}$ derived from FET the characteristics of Fig. 2 .

To characterize the SAW transmission along the delay line, we measured the $S_{21}$ scattering parameter, that is, the transmitted SAW intensity from one IDT to the other. In the upper panel of Fig. 4 a, we plot the variation of $S_{21}$ as a function of time. At $t=6 \mathrm{~s}$, a diffraction-limited spot in the centre of the hybrid device is irradiated for $\Delta t=5 \mathrm{~s}$ by either a red $(h v=1.87 \mathrm{eV})$ or an infrared laser $(h v=1.46 \mathrm{eV})$ source at $1 \mathrm{~mW}$ power. The red laser is resonant with the fundamental optical transition of $\mathrm{MoS}_{2}$ on $\mathrm{LiNbO}_{3}$, while the photon energy of the infrared laser is less than the optical bandgap of $\mathrm{MoS}_{2}$. We resolve a pronounced photoresponse for the red laser, manifesting itself in a reduction of the transmitted SAW signal $\left(\Delta S_{21}<0\right)$. Such an increase of the attenuation is expected from equation (1) since photogeneration of electrons and holes leads to an increase of $G$, while still remaining in the $G \ll G_{\mathrm{m}}$ regime. For the infrared laser, no variation of the SAW attenuation was resolved, corroborating that the observed response indeed arises from photogenerated carriers in the $\mathrm{MoS}_{2}$. We confirm this interpretation by simultaneously measuring the electrical conductivity in 2-point configuration, which is plotted in the lower panel of Fig. $4 \mathrm{a}$. The anticipated increase of the conductance $(\Delta G>0)$ is clearly resolved. The agreement of the global features of $\Delta S_{21}$ and $\Delta G$ is remarkable: both channels show quasi-instantaneous responses as the laser is switched on and off, which is attributed to the presence or absence of photogenerated carriers in the $\mathrm{MoS}_{2}$ layer. The associated processes occur on a timescale faster than the acquisition time of each data point of $250 \mathrm{~ms}$. In addition to this fast contribution, a response on longer (seconds to minutes) ${ }^{50}$ timescales is resolved clearly.

In Fig. 4 b, we present a detailed optical pump power series. In this experiment, the laser source is repeatedly switched on for $5 \mathrm{~s}$ every minute. The optical pump power is initially increased in $\Delta P_{\text {laser }}=0.1 \mathrm{~mW}$ steps from $P_{\text {laser }}=0.1-1 \mathrm{~mW}$ and then reduced to $P_{\text {laser }}=0$. The corresponding optical power pattern is plotted in the lower panel of Fig. 4b. The upper and centre panels compare the measured SAW $\left(\Delta S_{21}\right)$ and current $\left(I_{\mathrm{SD}}\right)$ responses for $V_{\mathrm{SD}}=+100 \mathrm{mV}$ and $-100 \mathrm{mV}$, respectively. Clearly, both the SAW attenuation and the FET current scale with the laser power in a nonlinear manner similar to the observations of Yin et $a l .{ }^{49}$ and Lopez-Sanchez et al. ${ }^{51}$ but different from the shortchannel devices of ref. 50. While the sign of $I_{\mathrm{SD}}$ depends on the polarity of $V_{\mathrm{SD}}, \Delta S_{21}$ decreases irrespective of the $V_{\mathrm{SD}}$ polarity. Furthermore, the amplitude of $\Delta S_{21}$ only depends on $P_{\text {laser }}$ and is independent of the applied $V_{\mathrm{SD}}$. These facts prove that electroacoustic and FET operation do not interfere. The component of the photoresponse with the longer time constant ${ }^{50}$ leads to the accumulation of higher sheet conductivity over the full 20-min duration of the experiment. Such processes are frequently observed in 2D materials and are typically attributed to traps at the interface to the substrate or in the material itself. In ref. 50, we demonstrate the composition dependence of this phenomenon for $\mathrm{MoS}_{2(1-x)} \mathrm{Se}_{2 x}$ alloys.

\section{Discussion}

Direct growth of $\mathrm{MoS}_{2}$ onto the $128^{\circ} \mathrm{YX}$-cut of $\mathrm{LiNbO}_{3}$ permits acousto-electric spectroscopy on the TMD overlayer as validated by the hybrid device assembled in this work. This finding opens many new avenues of research: while our hybrid device relied on metal contacts to the TMD film so as to validate congruence between electric transport and SAW-based conductivity measurements, subsequent experiments may dispense with the contacts, 
thereby allowing entirely contact-free transport measurements on TMD films. Moreover, the tight coupling of the TMD film to the underlying substrate as being indicated by the blueshift of the PL signal suggests that not only acousto-electric but also acoustomechanic spectroscopy on TMD films may be possible. In such experiments, the SAW exerts tensile or compressive strain allowing measurement of the coupling of the dynamic deformation to the electronic degrees of freedom of the TMD material. Spin and charge excitations in recently discovered TMD-based quantum $\operatorname{dots}^{53}$ could also be controlled dynamically by SAWdriven deformation potential coupling and Stark effect ${ }^{54}$. The rigid connection of the TMD layer to the $\mathrm{LiNbO}_{3}$ is crucial for
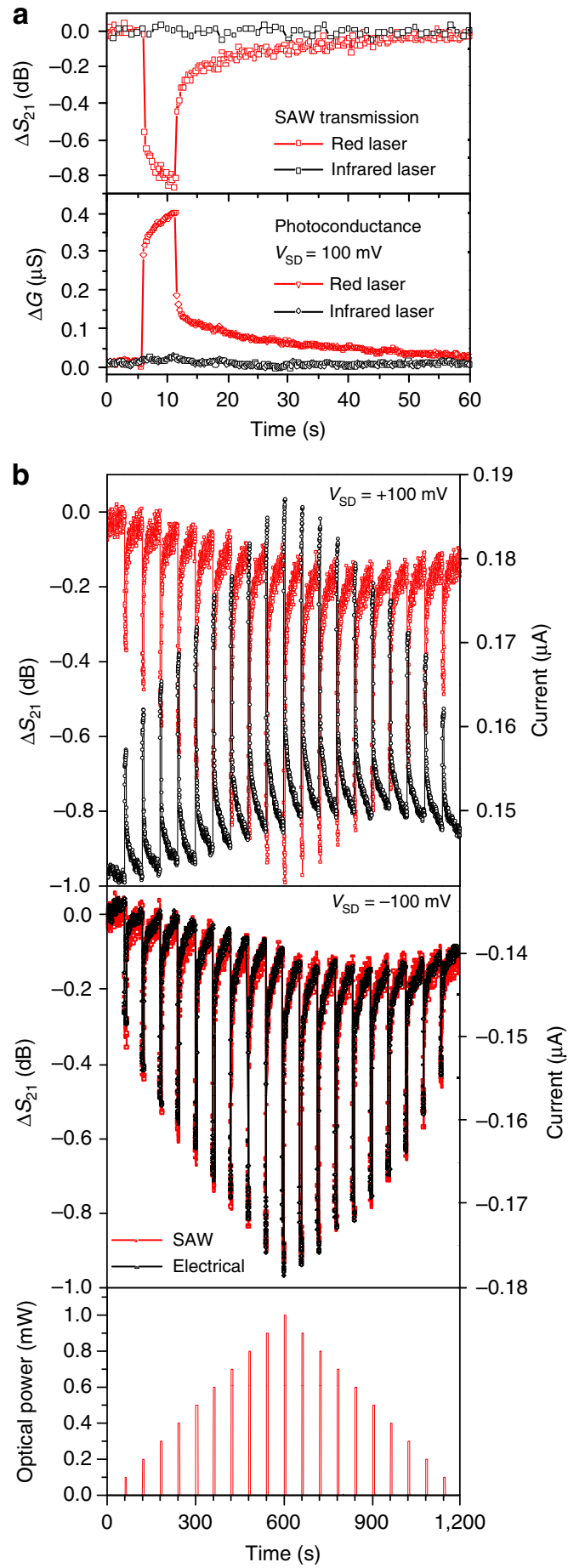

such acousto-mechanically driven approaches, since it ensures close coupling of the SAW to the film. We also highlight that our device fabrication used exclusively scalable techniques avoiding transfer or exfoliation steps. This paves the road towards the incorporation of TMD films as, for example, optically active elements, into conventional and inexpensive $\mathrm{LiNbO}_{3}$-based SAW devices of a type similar to those currently used, for example, as frequency filters in cell phones. As a consequence, we foresee that the fundamental device concept introduced in this article will attain widespread application both in the fundamental study of the properties of TMD films and in the technological realm where optically active thin, inorganic and durable films are desired: our SAW device remained functional for 9 months in air withstanding multiple intermittent thermal cycles of heating to temperatures as high as $>450 \mathrm{~K}$ and cooling to as low as $<10 \mathrm{~K}$ in vacuum in the meantime. Measurements on different TMD materials show promising initial results and will be reported on once completed.

\section{Methods}

Sample fabrication. TMD growth. Single-layer $\mathrm{MoS}_{2}$ synthesis follows the technique outlined in ref. 25: in a typical setup, $25 \mathrm{mg}$ of molybdenum trioxide powder (99.99\%, Sigma-Aldrich) was placed in an alumina boat and centred in the tube furnace. The $\mathrm{LiNbO}_{3}$ substrate $\left(128^{\circ} \mathrm{YX}\right.$-cut, oxygen reduced and weakly conductive 'black' $\mathrm{LiNbO}_{3}$, thickness $\left.d_{\text {sub }}=500 \mu \mathrm{m}\right)$ was mounted with a molybdenum mesh (Alfa Aesar) on the edge of the boat and $1 \mathrm{~g}$ of sulfur was placed upstream at a distance of $25 \mathrm{~cm}$ from the centre. The furnace was heated at a rate of $12.5^{\circ} \mathrm{C} \mathrm{min}{ }^{-1}$, held at $\sim 650{ }^{\circ} \mathrm{C}$ for $20 \mathrm{~min}$ and then allowed to cool naturally to room temperature. $\mathrm{N}_{2}$ carrier gas aided the transfer of the sulfur vapour to the sample region for optimal growth.

Device layout and fabrication. After PL identification of the region with monolayer growth, we fabricated Ti/Au metal contacts and IDTs (split-2 design, 21 finger pairs, duty cycle $1: 1$, aperture $200 \mu \mathrm{m}$ ) for electric and acoustic interfacing to the $\mathrm{MoS}_{2}$ film, respectively. The IDT's design wavelength was chosen as $\lambda_{\mathrm{SAW}}=25 \mu \mathrm{m}$, corresponding to a design frequency of $f_{\mathrm{SAW}}=160 \mathrm{MHz}$. Supplementary Fig. 5 shows a micrograph of an IDT. As shown in Fig. 1a, the two IDTs are located at opposite ends of the substrate in a delay line configuration (length $5.4 \mathrm{~mm}$ ) to enable us to launch and record SAWs propagating at

$v_{\mathrm{SAW}}=3,980 \mathrm{~m} \mathrm{~s}^{-1}$ in opposite directions across the chip. The studied FET device features a channel length of $L=35 \mu \mathrm{m}$ and a total width of the conducting channel of $W=360 \mu \mathrm{m}$. After fabrication of the electrical contacts, in a second lithographic step, an oxygen plasma treatment is used to remove the $\mathrm{MoS}_{2}$ single-layer film from the channel area except for a $180-\mu \mathrm{m}$-wide region across, which the SAW propagates. Thus, we ensure correspondence of the electrically and acoustically addressed film area. Figure 1c shows a PL map of the contact region after electrode deposition and removal of extraneous $\mathrm{MoS}_{2}$ film.

Lithographic patterning. All lithographic patterning of the IDTs and contact electrodes proceeded in a single exposure step using an electron beam writer and polymethyl methacrylate (PMMA) as resist. Contacts were fabricated by sequential deposition of $10 \mathrm{~nm}$ of Ti followed by $60 \mathrm{~nm}$ of $\mathrm{Au}$ in an e-beam evaporation tool. Subsequent lift-off defined the active structures. $\mathrm{MoS}_{2}$ was selectively removed in a

Figure 4 | Photoconductance spectroscopy. (a) Comparison of the timedependent photoresponse detected by the change of the transmitted SAW intensity $\left(\Delta S_{21}\right)$ with the change of the 2-point conductance $(\Delta G)$ of the FET. Red and black traces were recorded for $P_{\text {laser }}=1 \mathrm{~mW}$ excitation by a red and infrared laser, respectively. These lasers are switched on for $\Delta t=5 \mathrm{~s}$ at $t=6 \mathrm{~s}$. Both the instantaneous and persistent features of the photoresponse are consistently resolved by both measurement techniques. For excitation with an infrared laser, no photoresponse is detected, proving that the signal detected for the red laser indeed stems from the $\mathrm{MoS}_{2}$ monolayer. (b) Comparison of $\Delta S_{21}$ with $I_{S D}$ under photoexcitation using a red laser. The laser is switched on every $1 \mathrm{~min}$ for $\Delta t=5 \mathrm{~s}$. Each successive minute $P_{\text {laser }}$ is increased by $0.1 \mathrm{~mW}$ until $P_{\text {laser }}=1 \mathrm{~mW}$ is reached. Subsequently, $P_{\text {laser }}$ is decreased to $0 \mathrm{~mW}$ in steps of $\Delta P_{\text {laser }}=100 \mu \mathrm{W}$ as shown in the lower panel. Upper and centre panel compare the SAW transmission $\left(\Delta S_{21}\right.$, red) and photocurrent ( $I_{S D}$, black) for $V_{S D}=+100 \mathrm{mV}$ and $-100 \mathrm{mV}$, respectively. Direct correspondence between $\Delta S_{21}$ and $I_{S D}$ is confirmed: while $\Delta S_{21}$ reduces irrespective of voltages, the sign of $I_{S D}$ is determined by the polarity of $V_{S D}$. 
second e-beam exposure step and subsequent oxygen plasma treatment at a plasma power of $200 \mathrm{~W}$ at a pressure of $500 \mathrm{mTorr}$ for $13 \mathrm{~s}$.

Measurement techniques. SAW excitation and SAW transmission experiments. For measurement of the AEC and AEV, the output of an RF signal generator was amplified and connected to one of the IDTs. The RF characteristics of the IDTs and SAW transmission lines were characterized using a vector network analyser measuring scattering parameters of the RF network, in particular the scattering parameters $S_{11}$ (reflection) and $S_{21}$ (transmission and insertion loss).

Electrical characterization. 2-Point characterization was performed using a Keithley K2400 source meter unit (SMU). For 4-point characterization, a K2400 SMU was used only as a constant current source (no measurement probes connected) and the voltage at the potential probes was recorded directly by a K2000 digital multimeter. The gate voltage was applied by a Keithley K2600 SMU, which measured the gate leakage current at the same time. Short-circuit AEC and open-circuit AEV were recorded using a K2400 SMU with $V_{\mathrm{SD}}=0$ and $I_{\mathrm{SD}}=0$, respectively.

Optical spectroscopy. The photoconductivity experiments relied on red $(660 \mathrm{~nm})$ and infrared $(850 \mathrm{~nm})$ pulsed semiconductor lasers with a $80-\mathrm{MHz}$ repetition rate and a pulse duration $\leq 100 \mathrm{ps}$. PL and Raman spectroscopy, as well as mapping utilized a Horiba LabRAM HR spectroscopy system using a 532-nm excitation laser and a 1,800 lines per $\mathrm{mm}$ grating.

\section{References}

1. Weis, R. S. \& Gaylord, T. K. Lithium niobate: Summary of physical properties and crystal structure. Appl. Phys. A: Mater. Sci. Process. 37, 191-203 (1985).

2. Colin Campbell. Surface Acoustic Wave Devices and Their Signal Processing Applications xiv, 470 (Academic Press, 1989).

3. Ruppel, C. C. W., Reindl, L. \& Weigel, R. SAW devices and their wireless communications applications. IEEE Microw. Mag. 3, 65-71 (2002).

4. Arizmendi, L. Photonic applications of lithium niobate crystals. Phys. Status Solidi A 201, 253-283 (2004).

5. Kosters, M., Sturman, B., Werheit, P., Haertle, D. \& Buse, K. Optical cleaning of congruent lithium niobate crystals. Nat. Photon. 3, 510-513 (2009).

6. Jin, H. et al. On-chip generation and manipulation of entangled photons based on reconfigurable lithium-niobate waveguide circuits. Phys. Rev. Lett. 113, 103601 (2014)

7. Bhatt, R. et al. Urbach tail and bandgap analysis in near stoichiometric $\mathrm{LiNbO}_{3}$ crystals. Phys. Status Solidi A 209, 176-180 (2012).

8. Radisavljevic, B., Radenovic, A., Brivio, J., Giacometti, V. \& Kis, A. Single-layer $\mathrm{MoS}_{2}$ transistors. Nat. Nanotechnol. 6, 147-150 (2011).

9. Ghatak, S., Pal, A. N. \& Ghosh, A. Nature of electronic states in atomically thin $\mathrm{MoS}_{2}$ field-effect transistors. ACS Nano 5, 7707-7712 (2011).

10. Late, D. J. et al. Hysteresis in single-layer $\mathrm{MoS}_{2}$ field effect transistors. ACS Nano 6, 5635-5641 (2012).

11. Bao, W. Z., Cai, X. H., Kim, D., Sridhara, K. \& Fuhrer, M. S. High mobility ambipolar $\mathrm{MoS}_{2}$ field-effect transistors: substrate and dielectric effects. Appl. Phys. Lett. 102, 042104 (2013).

12. Mak, K. F. et al. Atomically thin MoS2: a new direct-gap semiconductor. Phys. Rev. Lett. 105, 136805 (2010).

13. Splendiani, A. et al. Emerging photoluminescence in monolayer $\mathrm{MoS}_{2}$. Nano Lett. 10, 1271-1275 (2010).

14. Le, D. et al. Spin-orbit coupling in the band structure of monolayer $\mathrm{WSe}_{2}$ J. Phys. Condens. Matter 27, 182201 (2015).

15. Kang, J., Tongay, S., Zhou, J., Li, J. B. \& Wu, J. Q. Band offsets and heterostructures of two-dimensional semiconductors. Appl. Phys. Lett. 102, $012111(2013)$

16. Mak, K. F., He, K. L., Shan, J. \& Heinz, T. F. Control of valley polarization in monolayer $\mathrm{MoS}_{2}$ by optical helicity. Nat. Nanotechnol. 7, 494-498 (2012).

17. Cao, T. et al. Valley-selective circular dichroism of monolayer molybdenum disulphide. Nat. Commun. 3, 887 (2012).

18. Lee, K., Gatensby, R., Mcevoy, N., Hallam, T. \& Duesberg, G. S. Highperformance sensors based on molybdenum disulfide thin films. Adv. Mater. 25, 6699-6702 (2013).

19. Nguyen, A. et al. Toward ferroelectric control of monolayer $\mathrm{MoS}_{2}$. Nano Lett. 15, 3364-3369 (2015).

20. Lee, H. S. et al. $\mathrm{MoS}_{2}$ nanosheets for top-gate nonvolatile memory transistor channel. Small 8, 3111-3115 (2013).

21. Bertolazzi, S., Krasnozhon, D. \& Kis, A. Nonvolatile memory cells based on $\mathrm{MoS}_{2}$ /graphene heterostructures. ACS Nano 7, 3246-3252 (2013).

22. Wang, H. et al. Integrated circuits based on bilayer $\mathrm{MoS}_{2}$ transistors. Nano Lett. 12, 4674-4680 (2012).

23. Van Der Zande, A. M. et al. Grains and grain boundaries in highly crystalline monolayer molybdenum disulfide. Nat. Mater. 12, 554-561 (2013).

24. Robinson, J. T. et al. Electronic hybridization of large-area stacked graphene films. ACS Nano 7, 637-644 (2013).

25. Mann, J. et al. Facile growth of sub-millimeter scale monolayer $\mathrm{MoS}_{2}$ films on $\mathrm{SiO}_{2} /$ Si. Eur. Phys. J. B 86, 226 (2013).
26. Yu, Y. et al. Controlled scalable synthesis of uniform, high-quality monolayer and few-layer $\mathrm{MoS}_{2}$ films. Sci. Rep. 3, 1866 (2013).

27. Wu, S. F. et al. Vapor-solid growth of high optical quality MoS2 monolayers with near-unity valley polarization. ACS Nano 7, 2768-2772 (2013).

28. Lee, Y.-H. et al. Synthesis of large-area $\mathrm{MoS}_{2}$ atomic layers with chemical vapor deposition. Adv. Mater. 24, 2320-2325 (2012).

29. Kang, K. et al. High-mobility three-atom-thick semiconducting films with wafer-scale homogeneity. Nature 520, 656-660 (2015).

30. Mann, J. et al. 2-Dimensional transition metal dichalcogenides with tunable direct band gaps: $\mathrm{MoS}_{2(1-\mathrm{x})} \mathrm{Se}_{2 \mathrm{x}}$ monolayers. Adv. Mater. 26, 1399-1404 (2014).

31. Weinreich, G., Sanders, T. M. \& White, H. G. Acoustoelectric effect in n-type germanium. Phys. Rev. 114, 33-44 (1959).

32. Parmenter, R. H. Acoustoelectric effect. Phys. Rev. 113, 102-109 (1959).

33. Hoskins, M. J., Morkoc, H. \& Hunsinger, B. J. Charge transport by surface acoustic waves in GaAs. Appl. Phys. Lett. 41, 332-334 (1982).

34. Rotter, M., Wixforth, A., Ruile, W., Bernklau, D. \& Riechert, H. Giant acoustoelectric effect in $\mathrm{GaAs} / \mathrm{LiNbO}_{3}$ hybrids. Appl. Phys. Lett. 73, 2128-2130 (1998).

35. Wixforth, A. et al. Surface acoustic waves on $\mathrm{GaAs}_{\mathrm{Al}} \mathrm{Ga}_{1-\mathrm{X}} \mathrm{As}$ heterostructures Phys. Rev. B 40, 7874-7887 (1989).

36. Rotter, M. et al. Single-chip fused hybrids for acousto-electric and acousto-optic applications. Appl. Phys. Lett. 70, 2097-2099 (1997).

37. Miseikis, V., Cunningham, J. E., Saeed, K., O'Rorke, R. \& Davies, A. G. Acoustically induced current flow in graphene. Appl. Phys. Lett. 100, 133105 (2012).

38. Bandhu, L., Lawton, L. M. \& Nash, G. R. Macroscopic acoustoelectric charge transport in graphene. Appl. Phys. Lett. 103, 133101 (2013).

39. Santos, P. V., Schumann, T., Oliveira, M. H., Lopes, J. M. J. \& Riechert, H. Acousto-electric transport in epitaxial monolayer graphene on SiC. Appl. Phys Lett. 102, 221907 (2013).

40. Poole, T., Bandhu, L. \& Nash, G. R. Acoustoelectric photoresponse in graphene. Appl. Phys. Lett. 106, 133107 (2015).

41. Pradhan, N. R. et al. Intrinsic carrier mobility of multi-layered $\mathrm{MoS}_{2}$ field-effect transistors on $\mathrm{SiO}_{2}$. Appl. Phys. Lett. 102, 123105 (2013)

42. Chen, J. R. et al. Control of Schottky barriers in single layer $\mathrm{MoS}_{2}$ transistors with ferromagnetic contacts. Nano Lett. 13, 3106-3110 (2013).

43. Yoon, Y., Ganapathi, K. \& Salahuddin, S. How good can monolayer $\mathrm{MoS}_{2}$ transistors be? Nano Lett. 11, 3768-3773 (2011).

44. Pignatiello, F. et al. Measurement of the thermal expansion coefficients of ferroelectric crystals by a Moire interferometer. Opt. Commun. 277, 14-18 (2007).

45. Okada, Y. \& Tokumaru, Y. Precise determination of lattice-parameter and thermal-expansion coefficient of silicon between $300-\mathrm{K}$ and 1500-K. J. Appl. Phys. 56, 314-320 (1984).

46. He, K., Poole, C., Mak, K. F. \& Shan., J. Experimental demonstration of continuous electronic structure tuning via strain in atomically thin $\mathrm{MoS}_{2}$. Nano Lett. 13, 2931-2936 (2013).

47. Hui, Y. Y. et al. Exceptional tunability of band energy in a compressively strained trilayer MoS2 sheet. ACS Nano 7, 7126-7131 (2013).

48. Radisavljevic, B. \& Kis., A. Mobility engineering and a metal-insulator transition in monolayer $\mathrm{MoS}_{2}$. Nat. Mater. 12, 815-820 (2013).

49. Yin, Z. et al. Single-layer $\mathrm{MoS}_{2}$ phototransistors. ACS Nano 6, 74-80 (2011).

50. Klee, V. et al. Superlinear composition-dependent photocurrent in CVD-grown monolayer $\mathrm{MoS}_{2(1-\mathrm{X})} \mathrm{Se}_{2 \mathrm{x}}$ alloy devices. Nano Lett. 15, 2612-2619 (2015).

51. Lopez-Sanchez, O., Lembke, D., Kayci, M., Radenovic, A. \& Kis, A. Ultrasensitive photodetectors based on monolayer $\mathrm{MoS}_{2}$. Nat. Nanotechnol. 8, 497-501 (2013).

52. Wixforth, A., Kotthaus, J. P. \& Weimann, G. Quantum oscillations in the surface acoustic wave attenuation caused by a two-dimensional electronsystem. Phys. Rev. Lett. 56, 2104-2106 (1986).

53. Chakraborty, C. et al. Voltage-controlled quantum light from an atomically thin semiconductor. Nat. Nanotechnol. 10, 507-511 (2015).

54. Weiss, M. et al. Dynamic acoustic control of individual optically active quantum dot-like emission centers in heterostructure nanowires. Nano Lett. 14, 2256-2264 (2014)

\section{Acknowledgements}

This work was supported by the US National Science Foundation (NSF) under grant no. ECCS-1435703, by the Deutsche Forschungsgemeinschaft (DFG) via the Emmy Noether Program (KR3790/2), by the Excellence Initiative of the German Federal Government via the Cluster of Excellence Nanosystems Initiative Munich (NIM) and by the BavariaCalifornia Technology Center (BaCaTeC). Additional support originates from C-SPIN, part of STARnet, a Semiconductor Research Corporation programme sponsored by MARCO and DARPA. E.P. and M.I. gratefully acknowledge fellowship support under DGE-1326120. 


\section{Author contributions}

L.B. and H.J.K. designed the research and coordinated the project. E.P. and F.J.R.S. prepared samples and preformed experiments assisted by A.E.N., D.B., M.I., G.v.S., I-H.L., W.M., B.M., J.M. and V.K. E.P. and F.J.R.S. analysed the data assisted by H.J.K., L.B. and A.W. H.J.K. and L.B. wrote the paper. All authors commented on the manuscript and approved the submitted version.

\section{Additional information}

Supplementary Information accompanies this paper at http://www.nature.com/ naturecommunications

Competing financial interests: The authors declare no competing financial interests.
Reprints and permission information is available online at http://npg.nature.com/ reprintsandpermissions/

How to cite this article: Preciado, E. et al. Scalable fabrication of a hybrid field-effect and acousto-electric device by direct growth of monolayer $\mathrm{MoS}_{2} / \mathrm{LiNbO}_{3}$. Nat. Commun . 6:8593 doi: 10.1038/ncomms9593 (2015).

(c) (i)

This work is licensed under a Creative Commons Attribution 4.0 International License. The images or other third party material in this article are included in the article's Creative Commons license, unless indicated otherwise in the credit line; if the material is not included under the Creative Commons license, users will need to obtain permission from the license holder to reproduce the material. To view a copy of this license, visit http://creativecommons.org/licenses/by/4.0/ 